\title{
ANÁLISE DE FORMULAÇÕES NPK FISCALIZADAS PELO MAPA, DE 2008 A 2010 $(1)$
}

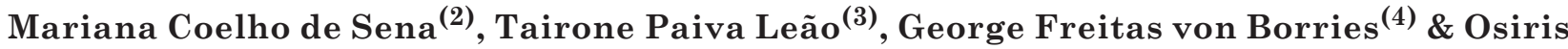 \\ Turnes ${ }^{(4)}$
}

\begin{abstract}
RESUMO
O Brasil ocupa o quarto lugar mundial em consumo de fertilizantes; dentre esses, destaca-se o fertilizante mineral misto como o mais consumido no País. Depois da atualização da legislação brasileira de fertilizantes em 2004, muitos produtores desses insumos afirmam que houve aumento excessivo no rigor da legislação em relação à fiscalização dos teores de nutrientes neles contidos. Dentro desse contexto, os objetivos deste trabalho foram aplicar técnicas de análise estatística exploratória e descritiva e de regressão logística aos dados de análises fiscais de fertilizantes realizadas pelo Ministério da Agricultura, Pecuária e Abastecimento (MAPA), nos anos de 2008 a 2010, buscando indicadores da contribuição das fontes de variação referentes aos fatores estabelecimento, formulação e laboratório para a variação total dos resultados; e verificar se os níveis de tolerância estabelecidos pelo MAPA estão sendo praticados. Para tanto, esses dados, separados por estabelecimento, formulação, laboratório, especificação granulométrica e período foram submetidos à análise descritiva, seguida de regressão logística. A regressão logística demonstrou que, para $\mathrm{N}$ e $\mathrm{P}$ analisados pelo laboratório com maior número de observações, as variáveis "estabelecimento" e "formulação" influem no resultado final da análise, dentro ou fora da garantia, enquanto para $\mathrm{K}$, analisado pelo mesmo laboratório, apenas a variável "formulação" influi nesses resultados.
\end{abstract}

Termos de indexação: fertilizante mineral misto, regressão logística, legislação.

(1) Parte da Dissertação de Mestrado da primeira autora. Recebido para publicação em 26 de agosto de 2013 e aprovado em 19 de maio de 2014.

(2) Fiscal Federal Agropecuário, Ministério da Agricultura, Pecuária e Abastecimento - MAPA. Esplanada dos Ministérios, bloco D, anexo A, sala 317. CEP 70043-900 Brasília (DF). E-mail: mariana.sena@agricultura.gov.br

(3) Professor, Faculdade de Agronomia e Medicina Veterinária, Universidade de Brasília - UnB. Campus Universitário Darcy Ribeiro, Ala Sul. Caixa Postal 4508. CEP 70910-960 Brasília (DF). E-mail: tleao@unb.br

(4) Professor, Departamento de Estatística, UnB. Campus Universitário Darcy Ribeiro. E-mail: gborries@unb.br; osiris@unb.br 


\title{
SUMMARY: ANALYSIS OF NPK FERTILIZERS INSPECTED BY MAPA FROM 2008 TO 2010
}

\begin{abstract}
Brazil is the fourth largest consumer of fertilizers in the world. Among these, bulk blends fertilizers are the most consumed in the country. After updating of Brazilian legislation in regard to fertilizers in 2004, many producers claim that there was an excessive increase in the strictness of legislation concerning inspection of nutrient levels in those products. Within this context, the objective of this study was to provide an exploratory and descriptive statistical analysis to the data gathered by MAPA (Ministry of Agriculture) from the inspection of the fertilizer industry from the years 2008 to 2010, looking for the most important source of variation, including producer, formula, and laboratory, and verifying if the minimum requirements established by law are being practiced. These data, separated by producer, formula, laboratory, particle size, and period, were subjected to descriptive analysis, followed by logistic regression. Logistic regression showed that for nitrogen and phosphorus analyzed by the laboratory with the greatest number of observations, the "producer" and "formula" variables affect the final results (not-in-conformity or in-conformity), whereas for potassium analyzed by the same laboratory, only the "formula" variable affected the results.
\end{abstract}

Index terms: bulk blend fertilizer, logistic regression, legislation.

\section{INTRODUÇÃO}

Segundo dados da Associação Nacional para Difusão de Adubos (ANDA), em 2009, foram entregues ao consumidor final 22.400.301 toneladas de fertilizantes, e cerca de $70 \%$ desses fertilizantes, e suas matérias-primas, são importados anualmente (ANDA, 2009). Entre 1994 e 2007, a taxa média de elevação da importação de fertilizantes foi de $9,83 \%$ ao ano, e o Brasil é o quarto maior consumidor mundial desses produtos (Saab \& Paula, 2008). Em razão do menor custo de produção, os fertilizantes minerais mistos, principalmente na forma de mistura de grânulos, ganharam espaço considerável no mercado brasileiro. Assim, a fiscalização desses insumos torna-se importante forma de controle para a manutenção da sua qualidade.

Define-se como fertilizante mineral misto, o produto resultante da mistura física de dois ou mais fertilizantes simples, complexos ou ambos (Brasil, 2004a). A média do índice de conformidade desses produtos foi de, aproximadamente, $82,5 \%$ entre 2004 e 2009, segundo dados contidos no site do Ministério da Agricultura, Pecuária e Abastecimento (MAPA). Em parte, a fiscalização de fertilizantes envolve sua coleta e análise química e física, com procedimentos definidos pela legislação pertinente. No caso da amostragem de fertilizantes, a Instrução Normativa (IN) MAPA n ${ }^{\circ} 10 / 2004$ estabelece a forma de coleta e a quantidade de produto a ser coletado (Brasil, 2004b). Aos resultados das análises fiscais, são admitidas ainda tolerâncias estabelecidas pela IN MAPA n ${ }^{0}$ 05/2007 (Brasil, 2007a). Assim, para o teor de macronutriente garantido de $6 \%$, admite-se até $5,4 \%$; para $20 \%$, até $19 \%$; e para $45 \%$, até $43,5 \%$ do nutriente.

São três as fontes de variação nos fertilizantes responsáveis pelas diferenças para menos em relação aos teores garantidos ou declarados: erro tecnológico, erro de amostragem e erro analítico (Malavolta, 1978).
Ainda segundo esse autor, o erro tecnológico é aquele que ocorre na preparação do produto, o analítico advém dos desvios decorrentes da análise laboratorial dos produtos e o de amostragem provém da coleta e do preparo do material. Dessa forma, no caso da fiscalização de fertilizantes, o erro analítico e o de amostragem são de responsabilidade do MAPA, justificando a existência das tolerâncias; e o tecnológico é de responsabilidade dos estabelecimentos produtores de fertilizantes.

A análise dos dados obtidos com a fiscalização de fertilizantes assume grande importância no direcionamento das ações fiscais e no controle da qualidade desses insumos fornecidos no mercado brasileiro, e, consequentemente, na manutenção da qualidade de solos e culturas. Dentro desse contexto, o objetivo deste trabalho foi fornecer uma análise exploratória e descritiva dos dados de análises fiscais de fertilizantes dos anos de 2008 a 2010 para subsidiar possíveis mudanças na legislação que rege a fiscalização de fertilizantes, com relação à tolerância e à divergência dos resultados analíticos.

\section{MATERIAL E MÉTODOS}

Foram utilizados, neste trabalho, dados compilados de 2008 a 2010, resultantes das análises fiscais realizadas pelos laboratórios do MAPA. Os dados resultam das análises fiscais das amostras coletadas em fiscalização de rotina do MAPA. As coletas foram feitas de acordo com os procedimentos da IN MAPA $n^{0}$ 10/2004 (Brasil, 2004b); o seu quarteamento foi realizado em quarteador do tipo Jones e a coleta foi realizada por fiscais federais agropecuários ou por representantes dos estabelecimentos fiscalizados sob a supervisão de fiscais. As amostras foram coletadas em estabelecimentos produtores, comerciantes e 
importadores. As análises física e química das amostras foram feitas seguindo métodos contidos na IN Secretaria de Defesa Agropecuária (SDA) n² 28/ 2007 (Brasil, 2007b).

Foram selecionados apenas os dados relativos à análise de fertilizantes minerais mistos sólidos para aplicação no solo, por representarem a maior parte do que se consome no País. Os laboratórios e os estabelecimentos produtores de fertilizantes foram codificados para tornar a análise imparcial. Os teores de N, P e K foram representados como formulações, na forma $\mathrm{N}-\mathrm{P}_{2} \mathrm{O}_{5}-\mathrm{K}_{2} \mathrm{O}$; o teor de $\mathrm{P}$ corresponde àquele solúvel em Citrato Neutro de Amônio mais água (CNA $+\mathrm{H}_{2} \mathrm{O}$ ). Assim, mantiveram-se informações sobre: $\mathrm{o}$ semestre em que o produto foi analisado; o estabelecimento responsável por sua produção; o laboratório responsável por sua análise fiscal; a quantidade de produto representada pela amostra coletada; a especificação granulométrica do produto, quando informada pela fiscalização; a formulação do produto; as garantias de $\mathrm{N}, \mathrm{P}_{2} \mathrm{O}_{5}$ solúvel em CNA + $\mathrm{H}_{2} \mathrm{O}$ e $\mathrm{K}_{2} \mathrm{O}$; os resultados das análises desses parâmetros e da especificação granulométrica; e o resultado final do certificado de análise de fiscalização.

Valendo-se da análise de frequência e com o auxílio dos gráficos de Pareto, foram determinadas as formulações mais frequentemente coletadas para análise fiscal e os estabelecimentos produtores com maior número de coletas. Para essas formulações, foram definidos a média, o erro-padrão, a mediana, a moda, a variância, o desvio-padrão, a amplitude, o valor mínimo e o valor máximo e o $1^{\circ}$ e $3^{\circ}$ quartis (Moore, 2000). A frequência de resultados de análises dentro da tolerância admitida e abaixo e acima dela foi calculada para todos os dados conjuntamente dividindo-se o número de valores dentro, abaixo ou acima do tolerado pelo total de observações. Posteriormente, foram calculadas as frequências, separadamente, por formulação (as mais frequentes), por estabelecimento produtor (os mais frequentes), por laboratório, por período e por especificação granulométrica. A tolerância foi calculada de acordo com o estabelecido pela IN MAPA n ${ }^{\circ}$ 05/2007 (Brasil, 2007a).

Para realizar a análise de regressão logística, selecionou-se o laboratório que continha o maior número de observações. Dentro desse laboratório, selecionaram-se apenas os estabelecimentos que correspondessem a pelo menos $2 \%$ dos dados e as formulações que correspondessem a pelo menos $1 \%$, para que houvesse dados suficientes para a realização da análise. A regressão logística é um caso especial de modelo linear generalizado, em que a componente aleatória para a variável resposta é binomial e a função de ligação é a transformação logito. Assim, a relação entre resposta (probabilidade de sucesso de um evento) e as variáveis resposta segue uma relação não linear monotônica na forma de uma curva em S (Hosmer \& Lemeshow, 2000). No caso deste trabalho, a variável resposta assume o valor 1 ("dentro"), para indicar que a amostra estava dentro da tolerância estabelecida pela IN MAPA n ${ }^{\circ}$ 05/2007, ou o valor 0 ("fora"), quando o resultado da análise fiscal indicava um valor acima ou abaixo do tolerado. A legislação não considera "fora" o resultado que se encontra acima do limite de tolerância.

Interessa calcular a probabilidade de $y$ ser igual a 1 , quando a variável independente é igual a $x$. Denotase essa probabilidade por $p(x)$, e ela é representada pela seguinte expressão:

$$
p(x)=\frac{e^{\beta_{0}+\beta_{1} x}}{1+e^{\beta_{0}+\beta_{1} x}}
$$

Neste trabalho, para a análise de regressão logística, foram consideradas como variáveis independentes: "estabelecimento", "período", "especificação granulométrica" e "formulação". O modelo logístico permitiu, a partir desses cálculos, verificar quais variáveis tinham influência significativa, para cada um dos macronutrientes estudados, utilizando os testes da razão de verossimilhança e de Wald. Posteriormente, foi testada a diferença entre os níveis de pares de variáveis independentes (Hosmer \& Lemeshow, 2000; Ott \& Longnecker, 2001). No caso da variável "formulação", considerou-se como "níveis" cada uma das formulações analisadas (por exemplo, 04-14-08 seria considerada um nível da variável "formulação" e 10-10-10 seria outro). As análises estatísticas foram realizadas utilizando-se o programa estatístico SAS, licenciado pela Universidade de Brasília.

\section{RESULTADOS E DISCUSSÃO}

\section{Estatística descritiva}

Utilizando-se a análise de frequência dos dados e os gráficos de Pareto, verificou-se que as 11 formulações que obtiveram maior número de observações foram, em ordem decrescente: 20-00-20 (389 observações), 04-14-08 (388 observações), 20-05-20 (366 observações), 02-20-20 (228 observações), 10-10-10 (183 observações), 05-20-20 (160 observações), 02-20-18 (157 observações), 08-28-16 (149 observações), 20-04-18 (133 observações), 08-20-20 (109 observações) e 20-00-10 (102 observações). Segundo Trani \& Trani (2011), as formulações mais encontradas no comércio para adubação de plantio são: 04-14-08, 08-28-16, 0530-10, 05-30-15, 04-20-20 e 05-25-25. Observou-se que duas das formulações mais comercializadas estão entre as 11 com maior número de dados de coleta, e uma é bastante próxima de uma das 11. Ainda segundo os autores citados, no caso das formulações com uso na adubação de cobertura, as mais encontradas são: 2005-20, 20-00-20, 20-05-15, 14-07-28, 12-06-12, 10-10-10 e 15-15-15. Pode-se concluir que as formulações coletadas pela fiscalização são também as mais 
comercializadas; ou seja, há boa representatividade na fiscalização do que é utilizado na agricultura brasileira. Quanto aos estabelecimentos com maior número de dados de coleta de amostra, têm-se aqueles codificados como 9.3, 3.7, 8.13, 9.4, 71.0, 18.0, 11.4, $11.2,8.5$ e 98.0 , em ordem decrescente.

Com relação à frequência de resultados dentro, acima e abaixo do tolerado, considerando-se todos os dados de fertilizantes minerais mistos, e apenas aqueles relativos aos macronutrientes primários $(\mathrm{N}, \mathrm{P}$ e K), obtiveram-se os resultados contidos no quadro 1.

Para esses nutrientes, no caso de fertilizantes minerais mistos sólidos para aplicação no solo, de acordo com a legislação vigente, valores acima da tolerância não são considerados infrações. Entretanto, para fins deste trabalho, considerou-se que esses valores também não são adequados, tendo em vista o desperdício de recurso não renovável e o possível prejuízo econômico associado. Assim, de acordo com o previsto em legislação, a frequência de conformidade para $\mathrm{N}$ é de $96,8 \%$; para $\mathrm{P}_{2} \mathrm{O}_{5}, 91,2 \%$; e para $\mathrm{K}_{2} \mathrm{O}$, $88,3 \%$, ou seja, em média, $92,1 \%$ dos fertilizantes minerais mistos analisados entre 2008 e 2010 estavam dentro das tolerâncias admitidas pela legislação. No entanto, ao se considerar que os valores acima do tolerado também estão fora do garantido, tem-se uma conformidade média de $66,4 \%$ para os macronutrientes primários dos fertilizantes minerais mistos.

Andrade (2004) obteve uma média de 20,9\% de fertilizantes fora das tolerâncias admitidas no Paraná. Essa diferença pode ser atribuída, segundo esse autor, ao fato de que os fertilizantes disponíveis no comércio (objeto de seu trabalho) estão mais sujeitos a variações causadas pela segregação do que aqueles coletados na indústria. Boa parte dos dados do MAPA refere-se a coletas realizadas na própria indústria. O K foi o nutriente com maior número de análises abaixo do tolerado, diferentemente do observado por Andrade (2004), que teve mais resultados abaixo do tolerado para P. As análises de frequência também foram feitas separadamente por estabelecimento, laboratório, período, formulação e especificação granulométrica. Para os 11 estabelecimentos com maior frequência de análise, os resultados estão apresentados na figura 1.

No caso do $\mathrm{N}$ e do $\mathrm{P}$, observou-se tendência de mais resultados acima do garantido do que abaixo da

Quadro 1. Análise de frequência de resultados de análise abaixo, dentro e acima do tolerado, para todos os dados de fertilizantes minerais mistos

\begin{tabular}{ccccc}
\hline Nutriente & Abaixo & Dentro & Acima & n \\
\cline { 2 - 4 } & \multicolumn{4}{c}{} \\
$\mathrm{N}$ & 3,2 & 67,9 & 28,9 & 7804 \\
$\mathrm{P}_{2} \mathrm{O}_{5}$ & 8,8 & 64,7 & 26,5 & 6631 \\
$\mathrm{~K}_{2} \mathrm{O}$ & 11,7 & 66,6 & 21,7 & 8197 \\
\hline
\end{tabular}

n: número de observações. tolerância; essa mesma observação feita por Andrade (2004) para N. As possíveis explicações são: os estabelecimentos tendem a colocar mais matéria-prima fonte do que o necessário; valem-se de um valor médio do teor de $\mathrm{N}$ da matéria-prima para o cálculo da formulação; e fazem uma compensação entre quantidade de matérias-primas mais caras e mais baratas. A hipótese do uso de valores médios torna-se mais provável no caso do $\mathrm{P}$, tendo em vista a maior variabilidade das matérias-primas utilizadas na fabricação de fertilizantes fosfatados. Ao contrário do observado neste trabalho, Andrade (2004) obteve teores de $\mathrm{P}$ menores que os garantidos, quando a análise foi feita por estabelecimento, para as misturas de grânulos no Estado do Paraná.

Verificou-se, para K, tendência diferente daquela observada para $\mathrm{Ne} \mathrm{P}$, resultado contrário àquele obtido por Andrade (2004) para misturas de grânulos. Esse resultado poderia indicar também que uma compensação com $\mathrm{N}$ pode ter ocorrido, tendo em vista que os resultados são opostos àqueles observados para esse nutriente. Outra possibilidade é a ocorrência de segregação, tendo em vista a diferença de tamanho dos grânulos que contêm $\mathrm{K}$ em relação àqueles que contêm N.

Quando as variáveis "laboratório" e "período" foram consideradas para a análise de frequência de conformidade, verificou-se, de maneira geral, a mesma tendência observada para todos os dados analisados conjuntamente: a frequência de resultados acima da
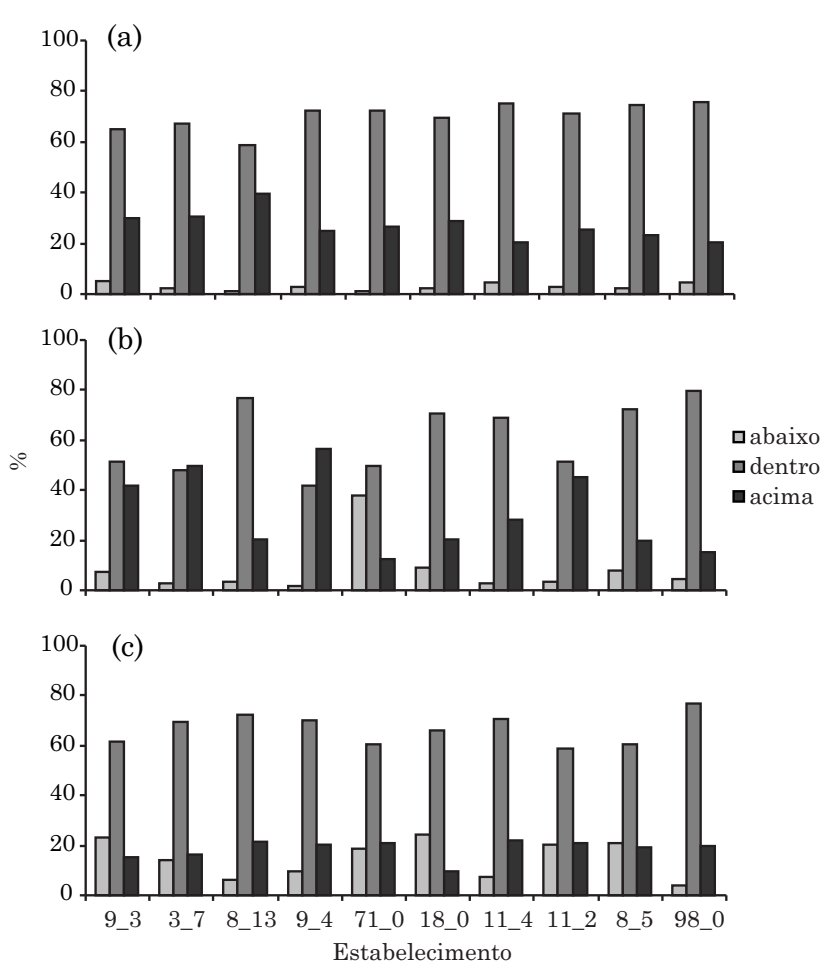

Figura 1. Frequências das conformidades das análises fiscais, por estabelecimento, para nitrogênio (a), fósforo (b) e potássio (c). 
tolerância é maior que aquela observada para os abaixo da tolerância.

Essa tendência repetiu-se também quando a análise foi feita por formulação, considerando-se apenas as 10 formulações mais frequentes. No caso do $\mathrm{N}$, notou-se que quanto menor o teor garantido, maior a tendência de obterem-se valores acima do tolerado (Figura 2). Andrade (2004) também observou que para a faixa de garantia de $\mathrm{N}$ entre 0 e $5 \%$, o índice de condenação dos produtos foi menor do que a média. No caso do $\mathrm{P}$ e do K, de maneira geral, foram obtidos mais resultados acima do tolerado, em comparação com aqueles abaixo do tolerado (Figura 2).

Quando as análises de frequência foram feitas considerando a especificação granulométrica, para $\mathrm{N}$, o maior número de resultados abaixo do tolerado ocorreu para os produtos classificados como "farelado fino" (Figura 3). Em razão da maior diferença entre o tamanho de partículas, que, como mencionado anteriormente, é fator que mais contribui para a ocorrência de segregação, esperava-se que produtos "sem especificação granulométrica" e "mistura de grânulos" obtivessem mais resultados abaixo do tolerado. Andrade (2004), porém, obteve maior frequência de inconformidades para a especificação "farelado" (26,4\%), seguido pela especificação mistura de grânulos (22,2 \%), quando analisou dados dos anos de 1997 a 2001, da fiscalização de fertilizantes no
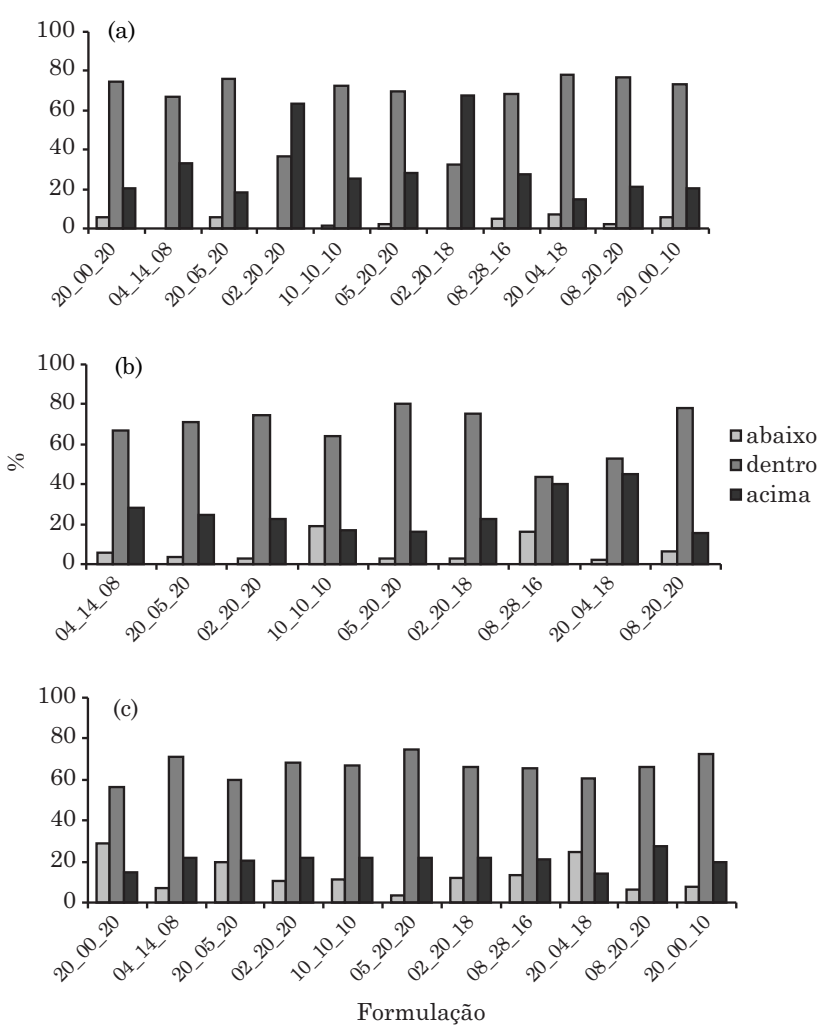

Figura 2. Frequências das conformidades das análises fiscais, por formulação, para o nitrogênio (a), fósforo (b) e potássio(c).
Estado do Paraná. No caso deste trabalho, no entanto, considerou-se que o número de dados, para as demais especificações, foi consideravelmente menor que o número de dados para "mistura de grânulos" e para "sem especificação", o que pode ter contribuído para os resultados.

Tanto para $\mathrm{P}$ como para $\mathrm{K}$, constatou-se que o número de observações abaixo e acima do tolerado é praticamente igual (Figura 3) para produtos "sem especificação granulométrica". A chance de segregação em um produto "sem especificação granulométrica" é, provavelmente, maior que aquela observada em outras especificações. Assim, considerando que, de acordo com Tosato (2006), a segregação pode causar tanto aumento como redução no teor de nutrientes, é possível que esses resultados tenham relação com esse fenômeno.

\section{Regressão logística}

$\mathrm{Na}$ análise de regressão logística para $\mathrm{N}$ e $\mathrm{P}$, verificou-se que apenas as variáveis "estabelecimento" e "formulação" foram consideradas significativas pelo modelo proposto. Tomando por base os resultados da análise de frequência realizados anteriormente, já era esperado que não houvesse efeito significativo para a variável "período". No caso da especificação granulométrica, como a maior parte dos dados referiase às especificações "mistura de grânulos" e "sem especificação", não se pode afirmar categoricamente que essa variável não tem influência na obtenção de resultados dentro e fora da garantia. A existência de poucos dados para as demais especificações pode ter influído nesse resultado.

Embora o modo de produção dos fertilizantes minerais mistos seja praticamente o mesmo para todos os misturadores, quanto à variável "estabelecimento",
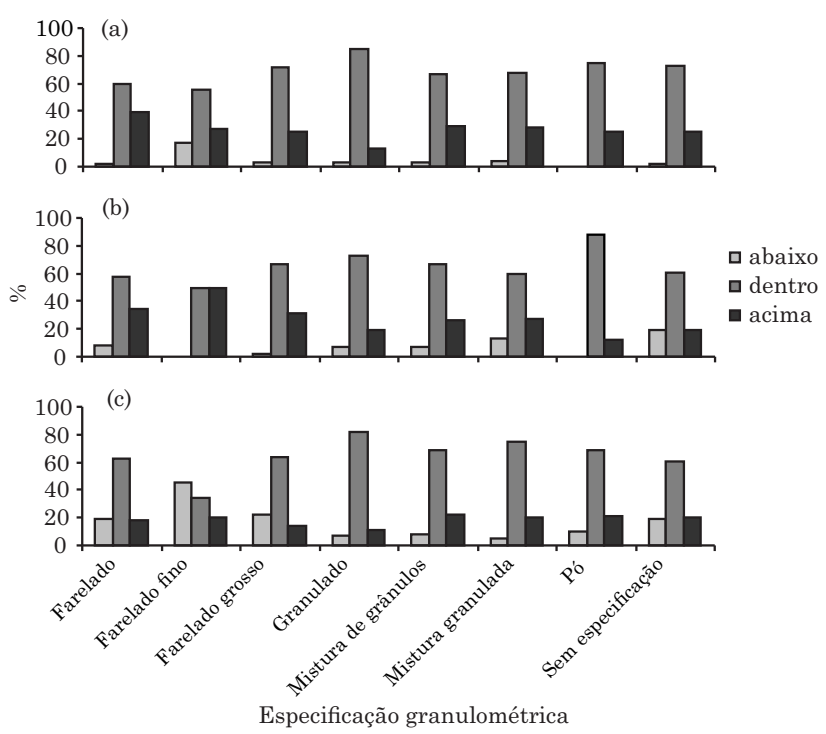

Figura 3. Frequências das conformidades das análises fiscais, por especificação granulométrica, para o nitrogênio (a), fósforo (b) e potássio (c). 
há diferenças no controle de qualidade de matériasprimas, calibração de balanças e manutenção de equipamentos. Além disso, no caso do $\mathrm{N}$ e do $\mathrm{P}$, é possível ocorrer o uso de diferentes matérias-primas, o que também poderia explicar essa diferença significativa entre estabelecimentos. No quadro 2, apresentam-se os resultados das comparações entre estabelecimentos dois a dois para $\mathrm{N}$.

O estabelecimento 30.1 chama a atenção pelo grande número de resultados significativos. Esses resultados indicam a razão de chances entre dois estabelecimentos, considerando-se como sucesso o elemento estar dentro da garantia. Assim, observouse que esse estabelecimento apresenta maior chance de obter um resultado dentro da garantia do que o estabelecimento com o qual é comparado, nos casos em que o resultado é significativo. Esse resultado pode indicar melhor controle por parte desse estabelecimento em relação aos demais.

No quadro 2, evidenciam-se os resultados da comparação entre estabelecimentos para P. Também nesse, chamam a atenção os resultados para o estabelecimento 30.1. Porém, o que se notou para esse nutriente é o inverso do observado para o $\mathrm{N}$; quando comparado com outros estabelecimentos, a chance de se obter um resultado dentro da garantia é menor.
No caso da diferença observada entre as formulações para $\mathrm{N}$, era esperada diferença significativa entre formulações, visto que houve tendência de maior número de resultados acima do garantido para teores mais baixos desse nutriente na formulação. No quadro 3, apresentam-se os resultados da razão de chances das formulações, comparadas duas a duas. Observou-se que parece haver alguma influência negativa do $\mathrm{P}$ sobre o $\mathrm{N}$, tendo em vista que as formulações que contêm aquele nutriente diferem, de maneira geral, daquelas que não o contêm. Esse fato revela chances menores de obtenção de resultados dentro da garantia para as formulações que contêm P. No caso das formulações, é provável que haja influência das matérias-primas fontes desse nutriente. Essas matérias-primas provêm de rochas, o que faz com que sua composição seja mais variável. Verificou-se que a formulação 20-05-20 apresentou maior chance de obtenção de resultados dentro da garantia (Quadro 4), em comparação com as formulações 04-14-08, 08-28-16 e 20-04-18, tendo em vista que a razão de chances obtida é menor que 1 .

$\mathrm{O}$ K comportou-se de maneira diferente do $\mathrm{N}$ e do P. O único efeito significativo para esse nutriente foi o da formulação. Os motivos para "período" e "especificação granulométrica" não serem significativos são provavelmente os mesmos que

Quadro 2. Razão de chances comparando-se estabelecimentos dois a dois para nitrogênio e fósforo

\begin{tabular}{|c|c|c|c|c|c|c|c|c|c|c|c|}
\hline & 11.2 & 18.0 & 18.1 & 30.1 & 3.20 & 3.7 & 8.5 & 8.7 & 9.3 & 9.4 & 9.8 \\
\hline & & \multicolumn{10}{|c|}{ Nitrogênio } \\
\hline 11.2 & 1 & & & & & & & & & & \\
\hline 18.0 & $1,439^{\mathrm{ns}}$ & 1 & & & & & & & & & \\
\hline 18.1 & $0,958^{\mathrm{ns}}$ & $0,666^{\mathrm{ns}}$ & 1 & & & & & & & & \\
\hline 30.1 & $0,236^{*}$ & $0,164^{*}$ & $0,246^{*}$ & 1 & & & & & & & \\
\hline 3.20 & $0,439^{\mathrm{ns}}$ & $0,305^{*}$ & $0,459^{\mathrm{ns}}$ & $1,861^{\mathrm{ns}}$ & 1 & & & & & & \\
\hline 3.7 & $0,795^{\mathrm{ns}}$ & $0,553^{*}$ & $0,830^{\mathrm{ns}}$ & $3,369^{*}$ & $1,810^{\mathrm{ns}}$ & 1 & & & & & \\
\hline 8.5 & $0,801^{\mathrm{ns}}$ & $0,557^{\mathrm{ns}}$ & $0,836^{\mathrm{ns}}$ & $3,391^{*}$ & $1,822^{\mathrm{ns}}$ & $1,007^{\mathrm{ns}}$ & 1 & & & & \\
\hline 8.7 & $0,450^{\mathrm{ns}}$ & $0,313^{\mathrm{ns}}$ & $0,470^{\mathrm{ns}}$ & $1,906^{\mathrm{ns}}$ & $1,024^{\mathrm{ns}}$ & $0,566^{\mathrm{ns}}$ & $0,562^{\mathrm{ns}}$ & 1 & & & \\
\hline 9.3 & $1,458^{\mathrm{ns}}$ & $1,013^{\mathrm{ns}}$ & $1,521^{\mathrm{ns}}$ & $6,137^{*}$ & $3,317^{*}$ & $1,832^{*}$ & $1,820^{\mathrm{ns}}$ & $3,239^{\mathrm{ns}}$ & 1 & & \\
\hline 9.4 & $0,362^{*}$ & $0,252^{*}$ & $0,378^{*}$ & $1,533^{\mathrm{ns}}$ & $0,823^{\mathrm{ns}}$ & $0,455^{\mathrm{ns}}$ & $0,452^{\mathrm{ns}}$ & $0,804^{\mathrm{ns}}$ & $0,248^{*}$ & 1 & \\
\hline \multirow[t]{2}{*}{9.8} & $1,574^{\mathrm{ns}}$ & $1,094^{\mathrm{ns}}$ & $1,642^{\mathrm{ns}}$ & $6,664^{*}$ & $3,580^{*}$ & $1,978^{\mathrm{ns}}$ & $1,965^{\mathrm{ns}}$ & $3,496^{\mathrm{ns}}$ & $1,080^{\mathrm{ns}}$ & $4,348^{*}$ & 1 \\
\hline & & & & & & Fósforo & & & & & \\
\hline 11.2 & 1 & & & & & & & & & & \\
\hline 18.0 & $0,603^{\mathrm{ns}}$ & 1 & & & & & & & & & \\
\hline 18.1 & $1,053^{\mathrm{ns}}$ & $1,745^{\mathrm{ns}}$ & 1 & & & & & & & & \\
\hline 30.1 & $3,716^{*}$ & $6,158^{*}$ & $3,529^{*}$ & 1 & & & & & & & \\
\hline 3.20 & $0,692^{\mathrm{ns}}$ & $1,147^{\mathrm{ns}}$ & $0,657^{\mathrm{ns}}$ & $0,186^{*}$ & 1 & & & & & & \\
\hline 3.7 & $1,804^{\mathrm{ns}}$ & $2,989^{*}$ & $1,713^{\mathrm{ns}}$ & $0,485^{\mathrm{ns}}$ & $2,605^{\mathrm{ns}}$ & 1 & & & & & \\
\hline 8.5 & $0,440^{\mathrm{ns}}$ & $0,730^{\mathrm{ns}}$ & $0,418^{\mathrm{ns}}$ & $0,119^{*}$ & $0,636^{\mathrm{ns}}$ & $0,244^{*}$ & 1 & & & & \\
\hline 8.7 & $0,843^{\mathrm{ns}}$ & $1,398^{\mathrm{ns}}$ & $0,801^{\mathrm{ns}}$ & $0,227^{\mathrm{ns}}$ & $1,218^{\mathrm{ns}}$ & $0,468^{\mathrm{ns}}$ & $1,915^{\mathrm{ns}}$ & 1 & & & \\
\hline 9.3 & $1,692^{\mathrm{ns}}$ & $2,804^{*}$ & $1,607^{\mathrm{ns}}$ & $0,455^{\mathrm{ns}}$ & $2,444^{\mathrm{ns}}$ & $0,938^{\mathrm{ns}}$ & $3,841^{*}$ & $2,006^{\mathrm{ns}}$ & 1 & & \\
\hline 9.4 & $1,267^{\mathrm{ns}}$ & $2,101^{\mathrm{ns}}$ & $1,204^{\mathrm{ns}}$ & $0,341^{\mathrm{ns}}$ & $1,831^{\mathrm{ns}}$ & $0,703^{\mathrm{ns}}$ & $2,877^{\mathrm{ns}}$ & $1,503^{\mathrm{ns}}$ & $0,749^{\mathrm{ns}}$ & 1 & \\
\hline 9.8 & $0,305^{\mathrm{ns}}$ & $0,505^{\mathrm{ns}}$ & $0,290^{\mathrm{ns}}$ & $0,082^{*}$ & $0,440^{\mathrm{ns}}$ & $0,169^{*}$ & $0,692^{\mathrm{ns}}$ & $0,361^{\mathrm{ns}}$ & $0,180^{*}$ & $0,241^{*}$ & 1 \\
\hline
\end{tabular}

${ }^{\mathrm{ns}}$ não significativo; * significativo a $5 \%$ pelo teste de Wald. Dados da coluna sobre dados da linha. 
aqueles indicados para $\mathrm{N}$ e $\mathrm{P}$. Quanto à variável "estabelecimento", todos os produtores utilizam, basicamente, o mesmo cloreto de potássio $(\mathrm{KCl})$ para a formulação dos fertilizantes minerais mistos, o que pode ter contribuído para esse resultado. Quanto ao resultado das comparações da razão de chances entre as formulações, observou-se que a formulação 10-0030 foi significativamente diferente de grande parte das formulações (Quadro 5). A chance de obter-se um resultado fora da garantia para essa formulação é

\section{Quadro 3. Razão de chances comparando-se formulações duas a duas para nitrogênio}

\begin{tabular}{|c|c|c|c|c|c|c|c|c|c|c|c|c|c|c|}
\hline & 04-14-08 & 08-24-12 & 08-28-16 & $10-00-30$ & $20-00-10$ & 20-00-15 & $20-00-20$ & 20-04-18 & 20-05-20 & $20-10-20$ & $25-00-25$ & $25-05-20$ & $30-00-10$ & $30-00-20$ \\
\hline 04-14-08 & 1 & & & & & & & & & & & & & \\
\hline $08-24-12$ & $1,400^{\mathrm{ns}}$ & 1 & & & & & & & & & & & & \\
\hline 08-28-16 & $1,367^{\mathrm{ns}}$ & $0,977^{\mathrm{ns}}$ & 1 & & & & & & & & & & & \\
\hline $10-00-30$ & $0,848^{\mathrm{ns}}$ & $0,606^{\mathrm{ns}}$ & $0,620^{\mathrm{ns}}$ & 1 & & & & & & & & & & \\
\hline $20-00-10$ & $0,252^{*}$ & $0,180^{*}$ & $0,184^{*}$ & $0,297^{*}$ & 1 & & & & & & & & & \\
\hline 20-00-15 & $0,099^{*}$ & $0,070^{*}$ & $0,072^{*}$ & $0,116^{*}$ & $0,391^{\mathrm{ns}}$ & 1 & & & & & & & & \\
\hline $20-00-20$ & $0,429 *$ & $0,306^{*}$ & $0,314^{*}$ & $0,505^{\mathrm{ns}}$ & $1,702^{\mathrm{ns}}$ & $4,350^{\mathrm{ns}}$ & 1 & & & & & & & \\
\hline $20-04-18$ & $0,301^{*}$ & $0,215^{*}$ & $0,220^{*}$ & $0,354^{*}$ & $1,193^{\mathrm{ns}}$ & $3,050^{\mathrm{ns}}$ & $0,701^{\mathrm{ns}}$ & 1 & & & & & & \\
\hline $20-05-20$ & $0,344^{*}$ & $0,246^{*}$ & $0,252^{*}$ & $0,406^{*}$ & $1,367^{\mathrm{ns}}$ & $3,493^{\mathrm{ns}}$ & $0,803^{\mathrm{ns}}$ & $1,145^{\mathrm{ns}}$ & 1 & & & & & \\
\hline $20-10-20$ & $0,347^{\mathrm{ns}}$ & $0,248^{*}$ & $0,254^{*}$ & $0,409^{\mathrm{ns}}$ & $1,379^{\text {ns }}$ & $3,523^{\mathrm{ns}}$ & $0,810^{\mathrm{ns}}$ & $1,155^{\mathrm{ns}}$ & $1,009^{\mathrm{ns}}$ & 1 & & & & \\
\hline $25-00-25$ & $0,515^{\mathrm{ns}}$ & $0,368^{*}$ & $0,377^{*}$ & $0,607^{\mathrm{ns}}$ & $2,045^{\mathrm{ns}}$ & $5,225^{*}$ & $1,201^{\mathrm{ns}}$ & $1,713^{\mathrm{ns}}$ & $1,496^{\mathrm{ns}}$ & $1,483^{\mathrm{ns}}$ & 1 & & & \\
\hline $25-05-20$ & $0,186^{*}$ & $0,133^{*}$ & $0,136^{*}$ & $0,219^{*}$ & $0,739^{\mathrm{ns}}$ & $1,889^{\mathrm{ns}}$ & $0,434^{\mathrm{ns}}$ & $0,619^{\mathrm{ns}}$ & $0,541^{\mathrm{ns}}$ & $0,536^{\mathrm{ns}}$ & $0,361^{\mathrm{ns}}$ & 1 & & \\
\hline $30-00-10$ & $0,404^{*}$ & $0,289^{*}$ & $0,295^{*}$ & $0,476^{\mathrm{ns}}$ & $1,604^{\mathrm{ns}}$ & $4,099^{\mathrm{ns}}$ & $0,942^{\text {ns }}$ & $1,344^{\mathrm{ns}}$ & $1,174^{\mathrm{ns}}$ & $1,164^{\mathrm{ns}}$ & $0,784^{\mathrm{ns}}$ & $2,170^{\mathrm{ns}}$ & 1 & \\
\hline $30-00-20$ & $0,579^{\mathrm{ns}}$ & $0,414^{\mathrm{ns}}$ & $0,424^{\mathrm{ns}}$ & $0,683^{\mathrm{ns}}$ & $2,300^{\mathrm{ns}}$ & $5,878^{*}$ & $1,351^{\mathrm{ns}}$ & $1,927^{\mathrm{ns}}$ & $1,683^{\mathrm{ns}}$ & $1,668^{\mathrm{ns}}$ & $1,125^{\mathrm{ns}}$ & $3,112^{\mathrm{ns}}$ & $1,434^{\mathrm{ns}}$ & 1 \\
\hline
\end{tabular}

${ }^{\text {ns }}$ não significativo; * significativo a $5 \%$ pelo teste de Wald. Dados da coluna sobre dados da linha.

Quadro 4. Razão de chances comparando-se formulações duas a duas para fósforo

\begin{tabular}{|c|c|c|c|c|c|c|c|}
\hline & 04-14-08 & 08-24-12 & $08-28-16$ & 20-04-18 & $20-05-20$ & $20-10-20$ & 25-05-20 \\
\hline 04-14-08 & 1 & & & & & & \\
\hline $08-24-12$ & $0,549^{\mathrm{ns}}$ & 1 & & & & & \\
\hline $08-28-16$ & $1,197^{\mathrm{ns}}$ & $2,182^{\mathrm{ns}}$ & 1 & & & & \\
\hline $20-04-18$ & $0,976^{\mathrm{ns}}$ & $1,780^{\mathrm{ns}}$ & $0,816^{\mathrm{ns}}$ & 1 & & & \\
\hline $20-05-20$ & $0,487^{*}$ & $0,887^{\mathrm{ns}}$ & $0,407^{*}$ & $0,498^{*}$ & 1 & & \\
\hline $20-10-20$ & $0,256^{\mathrm{ns}}$ & $0,468^{\mathrm{ns}}$ & $0,214^{*}$ & $0,263^{\mathrm{ns}}$ & $0,527^{\mathrm{ns}}$ & 1 & \\
\hline $25-05-20$ & $0,992^{\mathrm{ns}}$ & $1,809^{\mathrm{ns}}$ & $0,829^{\mathrm{ns}}$ & $1,017^{\mathrm{ns}}$ & $2,039^{\mathrm{ns}}$ & $3,870^{\mathrm{ns}}$ & 1 \\
\hline
\end{tabular}

ns não significativo; * significativo a $5 \%$ pelo teste de Wald. Dados da coluna sobre dados da linha.

\section{Quadro 5. Razão de chances comparando-se formulações duas a duas para potássio}

\begin{tabular}{|c|c|c|c|c|c|c|c|c|c|c|c|c|c|c|}
\hline & 04-14-08 & 08-24-12 & 08-28-16 & $10-00-30$ & 20-00-10 & 20-00-15 & 20-00-20 & 20-04-18 & 20-05-20 & $20-10-20$ & $25-00-25$ & 25-05-20 & $30-00-10$ & $30-00-20$ \\
\hline 04-14-08 & 1 & & & & & & & & & & & & & \\
\hline 08-24-12 & $1,115^{\mathrm{ns}}$ & 1 & & & & & & & & & & & & \\
\hline 08-28-16 & $1,032^{\mathrm{ns}}$ & $0,925^{\mathrm{ns}}$ & 1 & & & & & & & & & & & \\
\hline $10-00-30$ & $3,355^{*}$ & $3,008^{*}$ & $3,253^{*}$ & 1 & & & & & & & & & & \\
\hline $20-00-10$ & $0,968^{\mathrm{ns}}$ & $0,868^{\mathrm{ns}}$ & $0,939^{\text {ns }}$ & $0,289^{*}$ & 1 & & & & & & & & & \\
\hline $20-00-15$ & $1,230^{\mathrm{ns}}$ & $1,103^{\mathrm{ns}}$ & $1,193^{\mathrm{ns}}$ & $0,367^{\mathrm{ns}}$ & $1,270^{\mathrm{ns}}$ & 1 & & & & & & & & \\
\hline $20-00-20$ & $2,189^{*}$ & $1,963^{\mathrm{ns}}$ & $2,122^{*}$ & $0,652^{\mathrm{ns}}$ & $2,260^{*}$ & $1,779^{\mathrm{ns}}$ & 1 & & & & & & & \\
\hline 20-04-18 & $1,278^{\mathrm{ns}}$ & $1,146^{\mathrm{ns}}$ & $1,239^{\mathrm{ns}}$ & $0,381^{*}$ & $1,320^{\mathrm{ns}}$ & $1,039^{\mathrm{ns}}$ & $0,584^{*}$ & 1 & & & & & & \\
\hline $20-05-20$ & $1,733^{*}$ & $1,554^{\mathrm{ns}}$ & $1,680^{\mathrm{ns}}$ & $0,517^{\mathrm{ns}}$ & $1,790^{\mathrm{ns}}$ & $1,409^{\mathrm{ns}}$ & $0,792^{\mathrm{ns}}$ & $1,356^{\mathrm{ns}}$ & 1 & & & & & \\
\hline $20-10-20$ & $0,659^{\mathrm{ns}}$ & $0,591^{\mathrm{ns}}$ & $0,639^{\text {ns }}$ & $0,196^{*}$ & $0,681^{\mathrm{ns}}$ & $0,536^{\mathrm{ns}}$ & $0,301^{\mathrm{ns}}$ & $0,516^{\mathrm{ns}}$ & $0,380^{\mathrm{ns}}$ & 1 & & & & \\
\hline $25-00-25$ & $1,055^{\mathrm{ns}}$ & $0,945^{\mathrm{ns}}$ & $1,022^{\mathrm{ns}}$ & $0,314^{*}$ & $1,089^{\mathrm{ns}}$ & $0,857^{\mathrm{ns}}$ & $0,482^{*}$ & $0,825^{\mathrm{ns}}$ & $0,608^{\mathrm{ns}}$ & $1,600^{\mathrm{ns}}$ & 1 & & & \\
\hline $25-05-20$ & $0,989^{\mathrm{ns}}$ & $0,886^{\mathrm{ns}}$ & $0,958^{\text {ns }}$ & $0,295^{*}$ & $1,021^{\mathrm{ns}}$ & $0,804^{\mathrm{ns}}$ & $0,452^{\mathrm{ns}}$ & $0,773^{\text {ns }}$ & $0,570^{\mathrm{ns}}$ & $1,500^{\mathrm{ns}}$ & $0,938^{\text {ns }}$ & 1 & & \\
\hline $30-00-10$ & $0,603^{\mathrm{ns}}$ & $0,540^{\mathrm{ns}}$ & $0,584^{\mathrm{ns}}$ & $0,180^{*}$ & $0,622^{\mathrm{ns}}$ & $0,490^{\mathrm{ns}}$ & $0,275^{*}$ & $0,471^{\mathrm{ns}}$ & $0,348^{*}$ & $0,914^{\mathrm{ns}}$ & $0,571^{\mathrm{ns}}$ & $0,610^{\mathrm{ns}}$ & 1 & \\
\hline $30-00-20$ & $1,758^{\mathrm{ns}}$ & $1,576^{\mathrm{ns}}$ & $1,704^{\mathrm{ns}}$ & $0,524^{\mathrm{ns}}$ & $1,815^{\mathrm{ns}}$ & $1,429^{\mathrm{ns}}$ & $0,803^{\mathrm{ns}}$ & $1,375^{\mathrm{ns}}$ & $1,014^{\mathrm{ns}}$ & $2,667^{\mathrm{ns}}$ & $1,667^{\mathrm{ns}}$ & $1,778^{\mathrm{ns}}$ & $2,917^{*}$ & 1 \\
\hline
\end{tabular}

ns não significativo; * significativo a $5 \%$ pelo teste de Wald. Dados da coluna sobre dados da linha. 
maior, em comparação com a maioria delas. A diferença entre o tamanho dos grânulos que contêm $\mathrm{N}$ e aqueles com $\mathrm{K}$ pode ter alguma influência sobre esse resultado.

\section{CONCLUSÕES}

1. Não é possível afirmar que a tolerância não é adequada com base nos resultados deste trabalho.

2. De maneira geral, as amostras analisadas pelo MAPA entre 2008 e 2010 estão dentro dos limites de tolerância legal, estabelecidos para 11 formulações e para os nutrientes $\mathrm{N}, \mathrm{P}$ e K.

3. Pela análise de frequência, conclui-se que os 10 estabelecimentos analisados apresentam uma conformidade média de $92 \%$, se considerados os limites de tolerância legal apenas.

4. As garantias fornecidas de $\mathrm{N}$ tendem a ser subestimadas, em relação aos teores obtidos nas análises fiscais.

5. Quando analisados pelo laboratório com maior número de observações, a aceitação da mistura tem relação com o estabelecimento e a formulação, para $\mathrm{N}$ e P. Para K, há relação apenas com a formulação. Os poucos dados sobre especificação granulométrica não permitem concluir que esse fator não tem influência sobre o resultado da análise.

6. A presença de resultados fora da garantia para fertilizantes minerais mistos parece estar ligada a fontes de variação relacionadas não apenas à tolerância estabelecida pelo MAPA.

7. Os resultados são válidos apenas para o conjunto de dados analisado. Para obter informações mais amplas, faz-se necessária uma contínua análise dos dados da fiscalização ao longo dos próximos anos.

\section{AGRADECIMENTOS}

Aos Fiscais Federais Agropecuários que atuam na fiscalização de fertilizantes. Ao SAS Institute Brasil e Departamento de Estatística da Universidade de Brasília (UnB), pela parceria e cessão na utilização do software SAS.

\section{LITERATURA CITADA}

ASSOCIAÇÃO NACIONAL PARA DIFUSÃO DE ADUBOS ANDA. Preços vigentes - Vendas industriais (Mercado Brasileiro de Fertilizantes). In: Anuário Estatístico - Setor de Fertilizantes, 2009. CD-ROM
ANDRADE, R.R. Utilização de métodos estatísticos na fiscalização do comércio de fertilizantes no Estado do Paraná. Florianópolis, Universidade Federal de Santa Catarina, 2004. 135p. (Dissertação de Mestrado)

BRASIL. Ministério da Agricultura, Pecuária e Abastecimento. Instrução Normativa $n^{\circ}$ 05, de 23 fev. 2007. Aprova as definições e normas sobre as especificações e as garantias, as tolerâncias, o registro, a embalagem e a rotulagem dos fertilizantes minerais destinados à agricultura. Diário Oficial da República Federativa do Brasil, Brasília, 2007a. seção 1, p.10.

BRASIL. Ministério da Agricultura, Pecuária e Abastecimento. Instrução Normativa $n^{0} 28$, de 27 jul. 2007. Aprova os métodos analíticos oficiais para fertilizantes minerais, orgânicos, organominerais e corretivos. Diário Oficial da República Federativa do Brasil. Brasília, 2007b. seção 1, p.11.

BRASIL. Poder Executivo. Decreto n ${ }^{\circ}$ 4.954, de 14 jan. 2004. Aprova o regulamento da Lei $\mathrm{n}^{\circ} 6.894$, de 16 dez. 1980, que dispõe sobre a inspeção e fiscalização da produção e do comércio de fertilizantes, corretivos, inoculantes ou biofertilizantes destinados à agricultura, e dá outras providências. Diário Oficial da República Federativa do Brasil, Brasília, DF, 2004a. seção 1, p.2.

BRASIL. Ministério da Agricultura, Pecuária e Abastecimento. Instrução Normativa $n^{\circ} 10,6$ maio 2004. Aprova as disposições sobre a classificação e os registros de estabelecimentos e produtos, as exigências e critérios para embalagem, rotulagem, propaganda e para prestação de serviço, bem como os procedimentos a serem adotados na inspeção e fiscalização da produção, importação, exportação e comércio de fertilizantes, corretivos, inoculantes e biofertilizantes, destinados à agricultura. Diário Oficial da República Federativa do Brasil, Brasília, 2004b. seção 1, p.12.

HOSMER, D.W. \& LEMESHOW, S. Applied logistic regression. 2.ed. NewYork, John Wiley \& Sons, 2000. 375p.

MALAVOLTA, E. Fertilizantes: controle de qualidade. São Paulo, ANDA, 1978. 39p.

MOORE, D.S. A estatística básica e sua prática. Rio de Janeiro, LTC, 2000. 482p.

OTT, R.L. \& LONGNECKER, M. An introduction to statistical methods and data analysis. Belmont, Cengage Learning, 2001. 1283p.

SAAB, A.A. \& PAULA, R.A. O mercado de fertilizantes no Brasil: Diagnósticos e propostas de políticas. R. Polit. Agric., 2:5-24, 2008.

TRANI, P.E. \& TRANI, A.L. Fertilizantes: Cálculo de fórmulas comerciais. Campinas, Instituto Agronômico, 2011. 29p. (Boletim Técnico IAC, 208)

TOSATO, J.M.T. Segregação no transporte de fertilizantes comercializados em embalagens "Big Bag". Ponta Grossa, Universidade Estadual de Ponta Grossa, 2006. 115p. (Dissertação de Mestrado) 